\title{
Philip Drazin and Norman Riley: The Navier-Stokes equations : a classification of flows and exact solutions
}

\author{
London Mathematical Society Lecture Note Series, vol. 334, Cambridge University \\ Press, 2006, ISBN: 0-521-68162-6
}

Published online: 5 May 2011

(C) Springer-Verlag 2011

This is a unique and intriguing book, apparently completed by the second author after the sad death of the first author. It is the kind of book project that relatively few authors would feel comfortable taking on, yet most workers in the fluid mechanics community would find valuable both for research and teaching.

As is clear from the title, the book is a survey of exact solutions to the Navier-Stokes equations. The first task faced in writing such a survey is in the byline. How does one classify the exact solutions? The four chapter titles show how the authors have approached the task: Steady flows bounded by plane boundaries; Steady axisymmetric and related flows; Unsteady flows bounded by plane boundaries; Unsteady axisymmetric and related flows.

We find several familiar flows named for their discoverers: Couette and Poiseuille flow, of course, Hiemenz flow, Beltrami flows, Homann flow, Burgers' vortex, Ekman flow, and so on. The standard examples from many well-known texts, such as Kármán flow over a rotating disk and Hamel flow in a wedge, are treated but there is a great variety of refinements that will be known only to experts. The pace of development is brisk, and the reader will want to keep paper and pencil handy to check various steps as the exposition develops.

The book is tremendously useful both for the researcher searching for an analytically tractable case and for the instructor looking to spice up a lecture or pose a challenging problem to students. I doubt the book would be terribly successful as a text in a standard graduate course, unless it were a specialized course on exact solutions! As one reads through the various cases, one discovers a certain pattern of generalizations and refinements to the well-known basic flows. Walls can move in certain ways, and can become porous, but dramatic changes in geometry are typically not amenable to analysis. Nevertheless, I suspect most readers would be surprised at the richness of exact solutions and the many physical effects that these solutions reveal. Serious study of this text ought to precipitate changes in most viscous flow courses taught in universities worldwide. Although this short book is relatively expensive, I would warmly recommend it as an addition to any serious library on fluid mechanics. 